\title{
Prepectoral Breast Reconstruction: An Overview of the History, Technique, and Reported Complications
}

This article was published in the following Dove Press journal: Open Access Surgery

\author{
Joshua A Bloom (D) \\ Krishnabhai Patel (DD \\ Stephanie Cohen ${ }^{2}$ \\ Abhishek Chatterjee ${ }^{3}$ \\ Christopher Homsy ${ }^{4}$ \\ 'Department of Surgery, Tufts Medical \\ Center, Boston, MA, USA; ${ }^{2}$ Department \\ of Surgery, Tufts University School of \\ Medicine, Boston, MA, USA; ${ }^{3}$ Division of \\ Plastic Surgery, Tufts Medical Center, \\ Boston, MA, USA; ${ }^{4}$ Division of Plastic and \\ Reconstructive Surgery, Tulane University \\ Medical Center, New Orleans, LA, USA
}

Correspondence: Abhishek Chatterjee Department of Plastic Surgery, Chatterjee Tufts Medical Center, 800 Washington

Street, Boston, MA 021II, USA

Tel + 1860-874-7550

Fax + 1617-636-9095

Email Achatterjeel@tuftsmedicalcenter. org

\begin{abstract}
Prepectoral breast reconstruction is an increasingly prevalent form of breast reconstruction. Prepectoral breast reconstruction was abandoned in the past due to various complications but has recently made a resurgence due to the development of acellular dermal matrices and innovative techniques. The purpose of this review article is to discuss the history, techniques, benefits, and potential complications of prepectoral breast reconstruction The article also reviews current literature to evaluate published complication rates. Complications evaluated include infection $(3.67 \%)$, wound dehiscence $(2.10 \%)$, skin necrosis $(3.67 \%)$, seroma $(2.89 \%)$, hematoma $(1.34 \%)$, implant loss $(3.28 \%)$, return to the OR $(6.15 \%)$, contracture $(3.61 \%)$, and rippling $(7.38 \%)$. In conclusion, prepectoral breast reconstruction is a reliable, safe, and aesthetically feasible method of breast reconstruction. With increasing interest in this technique, it is important that surgeons and patients are familiar with prepectoral reconstruction and this article aims at providing this information.
\end{abstract}

Keywords: prepectoral, breast, reconstruction, trends, techniques, complications

\section{Introduction}

Breast cancer is the second most common cancer worldwide as of 2018 , according to the World Health Organization (WHO). With improved screening, advances in chemotherapy, targeted biologic therapy, and evolved surgical techniques, breast cancer survivorship has increased, allowing breast and plastic surgeons to focus on limiting surgical morbidity and increasing quality-of-life outcomes. While breast-conserving surgery and mastectomy with reconstruction are both oncologically safe options, breast-conserving surgery is sometimes contraindicated and safe oncologic breast surgery requires mastectomy. ${ }^{1}$ Implant-based reconstruction has been the most used technique for post-mastectomy breast reconstruction, ${ }^{2}$ mainly in the form of submuscular or dual plane (partial submuscular) implant placement. ${ }^{3}$ With the development of acellular dermal matrix (ADM) for soft tissue coverage, prepectoral breast reconstruction has become a more viable alternative. This review aims to discuss the history, techniques, benefits, and potential complications of prepectoral breast reconstruction.

\section{History of Prepectoral Breast Reconstruction and Trends of Reconstruction}

With the advent of silicone gel breast implants in the 1960s, prosthetic breast reconstruction became possible. These first implant-based breast reconstructions were performed by placement of the implant in a subcutaneous pocket directly underneath the mastectomy skin flap. This approach was relatively simple, quick, 
did not violate the integrity of the muscle, but was fraught with complications. Early prepectoral breast reconstruction was associated with implant malposition, visibility, palpability, rippling, implant exposure, and capsular contracture, among others. These complications were found to be the result of inadequate soft tissue coverage, and thus reconstruction techniques moved from prepectoral implant placement to submuscular (Figure 1). ${ }^{4,5}$

The submuscular technique places the implant under the pectoralis major muscle without releasing the inferior insertion of the muscle to the chest wall. Lateral and inferior coverage of the implant is obtained by mobilization of muscle flaps, serratus anterior, and rectus abdominis, respectively. ${ }^{4}$ This total muscular technique allows for vascularized soft tissue coverage of the implant while simultaneously decreasing the rate of capsular contracture compared to the subcutaneous placement. ${ }^{5}$ This was not without its drawbacks; however, as contraction of the muscle often resulted in pain due to muscle spasms as well as the phenomenon of animation deformity, where the implant is displaced upward and superolaterally with forceful pectoralis muscle contraction, causing a significant distortion of the breast mound. Moreover, the submuscular placement of the implant prevented lower pole expansion resulting in poor projection and definition of the breast. ${ }^{5,6}$

To overcome these limitations, the partial muscle coverage or dual-plane approach was developed. In this technique, the implant is covered partially by the pectoralis major muscle superiorly and by the mastectomy skin
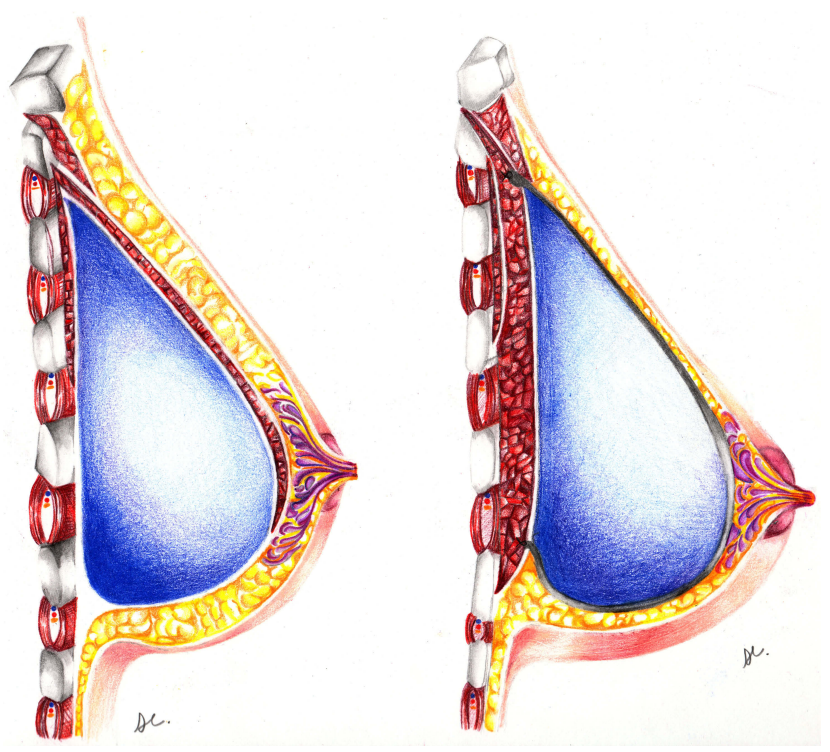

Figure I Partial submuscular breast reconstruction (left) compared with prepectoral breast reconstruction (right). flap only inferiorly. This technique allows for an improved lower pole expansion but results in a superior migration of inferiorly released pectoralis major muscle, creating a phenomenon known as "window-shading". 4 Additionally, the subcutaneous coverage at the lower pole and the absence of muscle support may cause the implant to stretch out the lower pole of the breast leading to an excessive inferior migration commonly referred to as "bottoming out".

With the introduction of ADMs in 2006, the modified dual-plane approach was pioneered. This technique utilized $\mathrm{ADM}$ as a sling to anchor the inferior edge of the pectoralis major to the inframammary fold and provide lateral support to better define the lateral mammary fold and mitigate the incidence of implant lateralization and inferior migration. 5,6 The use of ADM also allows for more tissue expansion compared to total submuscular coverage. In a study comparing total submuscular to subpectoral to partly subpectoralassisted ADM group, the authors found that intraoperative fill volumes were significantly higher (412 versus $130 \mathrm{~mL}$ ) and the total number of expansions prior to final expander to implant exchange was much less (1.7 versus 4.3 expansions) in the partly subpectoral-assisted ADM group compared to the total muscle coverage group. ${ }^{7}$

Many experts consider the dual-plane technique with or without ADM placement to be the accepted standard technique of implant-based reconstruction. However, this approach still carries its own drawbacks and set of complications. As with the subpectoral implant placement, elevation of the muscle can lead to animation deformity, chest tightness, pain, and muscle spasms. The treatment of these muscle related complications is changing the implant pocket from the subpectoral back to the prepectoral position. Therefore, due to the positive outcomes seen initially with these revision cases, there has now been a shift to performing primary prepectoral breast reconstruction. ${ }^{5,6}$

\section{Techniques for Prepectoral Breast Reconstruction}

The advances in both ADM and implant technology have made prepectoral breast reconstruction approach an attractive option from a reconstructive standpoint. ADM appears to decrease the rates of capsular contracture, ${ }^{3,4}$ results in a more natural, aesthetically pleasing breast shape, and increases the success rate of prepectoral breast reconstruction by providing another layer of soft tissue coverage between 
the implant and mastectomy skin flaps. Similarly, newer generation cohesive silicone implants may reduce implant rippling and visibility. ${ }^{5}$

A number of criteria need to be met for prepectoral breast reconstruction to be accomplished safely and appropriately. Immediate prepectoral implant is appropriate and has been described in the literature for both skin-sparing and nipple-sparing mastectomies. Skin-sparing mastectomy is utilized over nipple-sparing for cancers that infiltrate the nipple-areolar complex (NAC) or if the breast skin envelope was too large or ptotic to allow NAC preservation. Of note, in instances such as this, Scott Spear has described a 2-stage operation consisting of an initial reduction to decrease the skin envelope followed by a second-stage nipple-sparing mastectomy with reconstruction (either with direct to implant or tissue expander if the envelope is hypoperfused). ${ }^{8}$

Following mastectomy, whether skin or nipple-sparing, the first step begins with evaluating the quality and perfusion of the mastectomy skin flaps. Mastectomy flap perfusion is a key element to achieve a successful prepectoral reconstruction. This may be initially assessed by a thorough inspection of the skin flaps and the presence of a healthy subdermal fat layer although there is no "one size fits all" flap thickness, as subcutaneous tissue varies between patients based on race and age, among other variables. This is difficult to control given the variation among patients; however, perfusion is assessed with indocyanine green (ICG) angiography ICG if there are concerns. The advent of laser angiography has revolutionized plastic and reconstructive surgery and is now widely available ICG dye to assess flap perfusion (Figure 2). In our experience, the nipple is always hypoperfused after nipplesparing mastectomy when observed under ICG angiography but survives with subsequent epidermolysis. If the skin perfusion is compromised as well, it is then better to stage the reconstruction by first inserting tissue expanders in the prepectoral plane, to allow time for skin flaps to develop robust perfusion through the delay phenomenon for a period of 2 to 3 weeks. ${ }^{5}$

The goal of prepectoral breast reconstruction is to create a "hand-in-glove" fit with respect to the implant and pocket size. Thick mastectomy flaps, appropriately sized implants, and the use of cohesive silicone gel implants optimize this relationship. If the pocket is larger than the implant, it is more likely that rippling, wrinkling, and visibility will become an issue. ${ }^{5}$

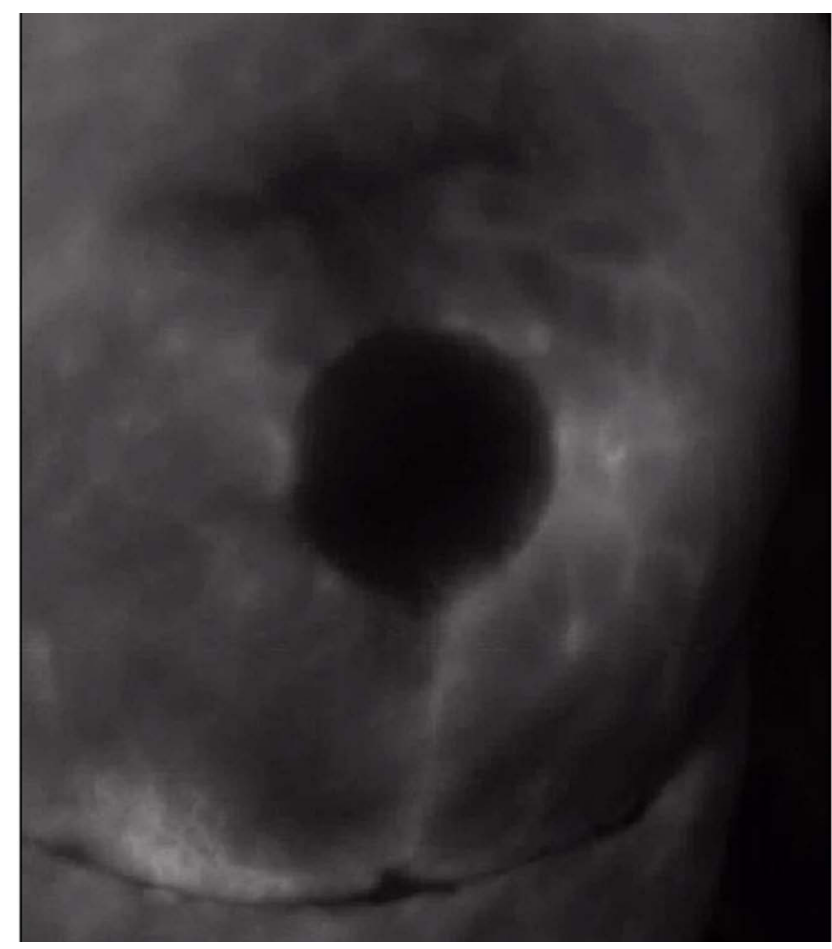

Figure 2 Representative indocyanine green laser angiography showing good soft tissue perfusion following nipple-sparing mastectomy and prepectoral reconstruction.

ADMs are used in the process of prepectoral breast reconstruction as they provide tissue support. The ADM is sized by draping it over the implant so that it conforms to the implant without any laxity. There are two main techniques related to the handling of ADM in prepectoral breast reconstruction. The first is an anterior wrap, which was originally the only "on-label" technique to provide implant support. The ADM is sutured to the superior border of the designed pocket followed by the medial and lateral borders. The implant is then inserted, and the ADM is sutured inferiorly. ${ }^{5,9}$ Alternatively, another approved approach involves wrapping the ADM completely around the implant or expander, as described by Sbitany et $\mathrm{al}^{3}$. The ADM is first shaped to the size of the implant/expander (Figure 3). A 2-3 cm cuff of ADM is sutured at the level of the desired inframammary fold (IMF) in 2 suture rows (Figure 4), the implant/expander is then placed on the surface of the pectoralis muscle and the ADM is wrapped around the device and sutured circumferentially (Figure 5). ${ }^{3,5,10}$ The complete wrap is not only more expensive owing to its use of a larger sheet of ADM but data have shown higher incidence of implant loss, capsular contracture, rippling, seroma, and flap necrosis. $^{11}$

The presence of ADM usually leads to seroma formation and thus one to two drains are typically placed in each breast. 


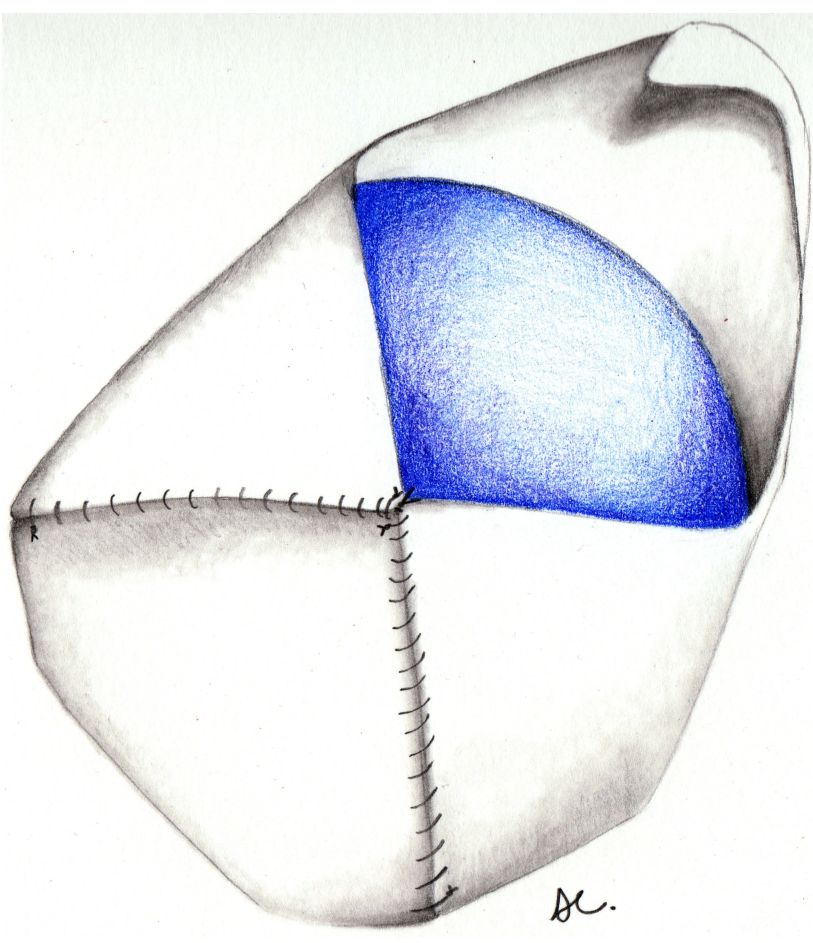

Figure 3 Acellular Dermal Matrix (ADM) sized and wrapped circumferentially around the implant/expander to create a pocket.

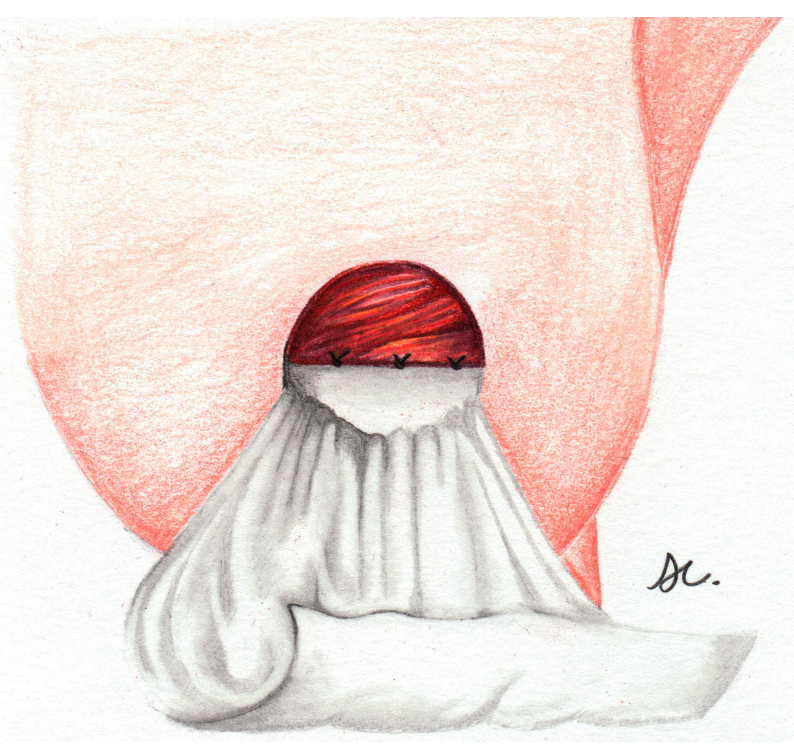

Figure 4 Cuff of Acellular Dermal Matrix (ADM) sutured at the level of the desired inframammary fold (IMF) in 2 suture rows following skin-sparing mastectomy.

The ADM is typically fenestrated to allow for more rapid integration and improved fluid drainage. If an expander is placed, expansion begins 2 to 3 weeks postoperatively and drains are left in place for a minimum of 3 weeks. Drains are removed once output is less than 20cc/day for 3 consecutive days. It should be mentioned that the second-stage expander to implant operation is performed through a new incision if

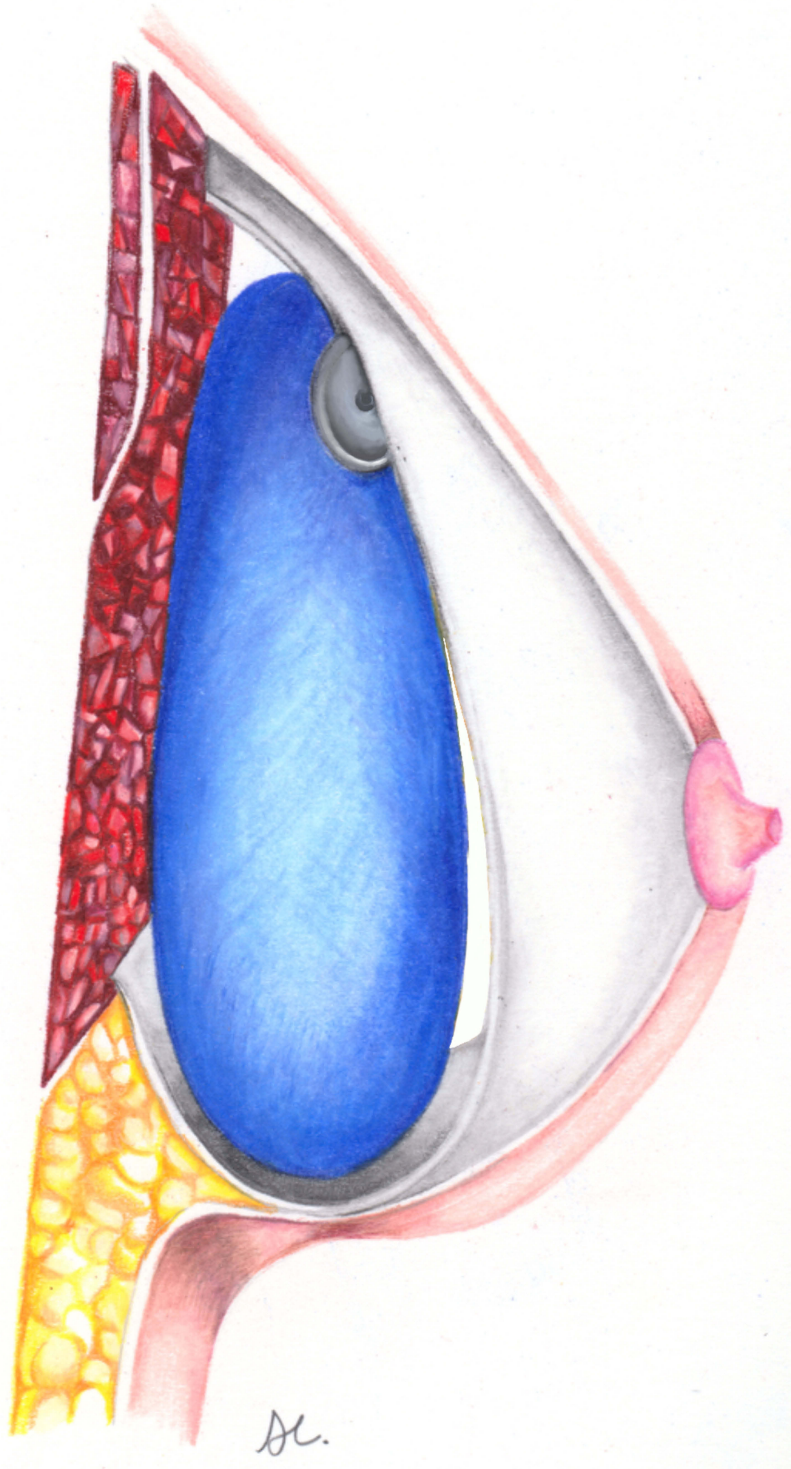

Figure 5 Prepectoral breast reconstruction with implant/expander placed on the surface of the pectoralis muscle and the Acellular Dermal Matrix (ADM) wrapped around the device and sutured circumferentially.

the tissue is irradiated. ${ }^{3}$ Of note, some authors choose to mesh ADM with a standard meshing machine. Compared to non-meshed ADM, meshed-ADM results in decreased fluid drainage, and shorter time to drain removal. ${ }^{12}$

\section{Prepectoral Breast Reconstruction Following Nipple-Sparing Mastectomy}

Nipple-sparing mastectomy surgical technique has improved patient-reported-outcomes with postmastectomy breast reconstruction while being oncologically safe. There is an intrinsic benefit to maintaining the complete external breast envelope 
for use in reconstruction. The traditional submuscular or dualplane techniques are still routinely used and offer vascularized soft tissue coverage but involve greater dissection and mobilization of the chest wall due to disinsertion and elevation of the pectoralis muscle during creating of the pocket. The placement of the implant in the prepectoral space avoids muscle dissection, limiting impaired muscle function, muscle spasms, postoperative pain, and the dreaded animation deformity. Additional surgery is needed to correct the animation deformity but converting the submuscular or dual plane implant to prepectoral. Thus, there has been an increasing trend to perform primary prepectoral breast reconstruction. ${ }^{3}$

Sbitany et al reviewed their experience and outcomes with two-stage prepectoral breast reconstruction compared to dual-plane breast reconstruction following nipple-sparing mastectomy. From 2012 to 2016, patients undergoing immediate breast reconstruction following nipple-sparing mastectomy were placed into either the dual-plane or prepectoral group based on the surgeon's intraoperative assessment of the skin flap perfusion. Demographics between the two groups were comparable and there were no statistical differences between groups (including diabetes, smoking, or follow-up time). In this cohort, there were no significant differences in complication rates between the two groups, including infection, seroma, and explantation rates. ${ }^{3}$ The major difference between the two groups was that the mean fill volume and the mean number of fills to complete expansion was higher in the submuscular group $(410 \pm 145 \mathrm{~mL}$ versus $371 \pm 125 \mathrm{~mL}$, and $4.4 \pm 1.9$ versus $3.1 \pm 1.2$, respectively) than in the prepectoral group. ${ }^{3}$

\section{Past Literature Reviews}

A comprehensive literature review looking at pre-pectoral implant reconstruction by Chatterjee et $\mathrm{al}^{13}$ further confirmed that the complication rate and safety profiles between the prepectoral and dual-plane groups were similar without any significant differences. The elimination of pectoralis muscle mobilization may make the postoperative recovery period and subsequent expansion process more comfortable, but this has not been proven in patient-reported outcome studies. In general, studies reviewing pre-pectoral breast reconstruction note that for prepectoral breast reconstruction to be successful, patient selection is key. ${ }^{13}$

\section{Complications}

As mentioned above, the prepectoral placement of the implant as compared to the submuscular approach avoids the complications associated with pectoralis major muscle dissection and mobilization. These include impaired functionality, muscle spasms, animation deformity, and "window-shading" among others. Nevertheless, the prepectoral approach is not without its drawbacks as well. ${ }^{14,15}$ The most common complication is capsular contracture with a rate of $8.8 \%$ in a recent systematic review of prepectoral breast reconstruction complications. Upon subgroup analysis, however, the rate of capsular contracture with the use of ADM was decreased to $2.3 \%$ as compared to $12.4 \%$ without. $^{15}$ While lending to lower capsular contracture and overall complications rates, the use of ADM was correlated with a higher rate of implant loss, infection, and mastectomy flap necrosis. ${ }^{15}$ ADM is also associated with red breast syndrome with an incidence of $6.4 \%{ }^{16}$ This entity is characterized by erythema directly overlying the ADM and is thought to be secondary to lymphedema and lymphatic obstruction postoperatively. All cases of breast erythema are empirically treated with antibiotics, but discontinued after 1 week if no change, and redbreast syndrome is presumed. Most cases are self-limiting, but prolonged red breast syndrome is occasionally treated with explantation of ADM and implant and conversion to autologous breast reconstruction. ${ }^{16}$

Other important complications to consider are infection, seroma, hematoma, implant loss, mastectomy flap necrosis, and NAC necrosis (Tables 1 and 2). It should be noted that long-term comparative studies between prepectoral and subpectoral implant placement complications are not yet available. There are many studies, mostly retrospective, that have published complication rates with prepectoral reconstruction. These rates were reviewed through a literature review searching for articles pertaining to prepectoral reconstruction on PubMed. Twenty-eight

Table I Complications of Prepectoral Breast Reconstruction Reported in the Literature (Average Follow-Up Was 19 Months (Range 4-55 Months)). ${ }^{19-46}$

\begin{tabular}{|l|l|}
\hline Complication & Pooled Rate (\%) \\
\hline Surgical site infection & $3.67 \%$ \\
Wound dehiscence & $2.10 \%$ \\
Skin necrosis & $3.67 \%$ \\
Seroma & $2.89 \%$ \\
Hematoma & $1.34 \%$ \\
Implant exposure/loss & $3.28 \%$ \\
Return to the OR & $6.15 \%$ \\
Contracture & $3.61 \%$ \\
Rippling & $7.38 \%$ \\
Any complication & $8.71 \%$ \\
\hline
\end{tabular}




\begin{tabular}{|c|c|}
\hline 宗㝘 & 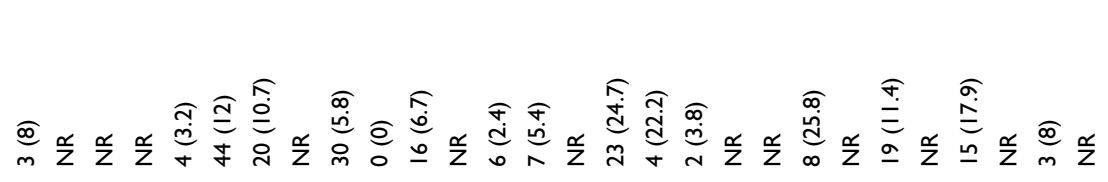 \\
\hline 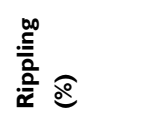 & 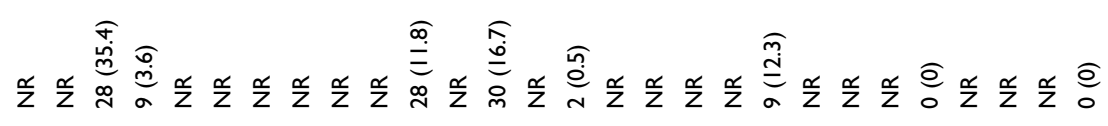 \\
\hline 仓ัँ & 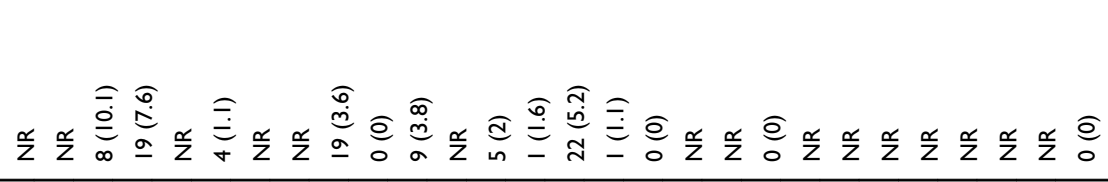 \\
\hline 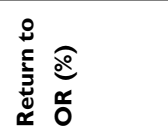 & 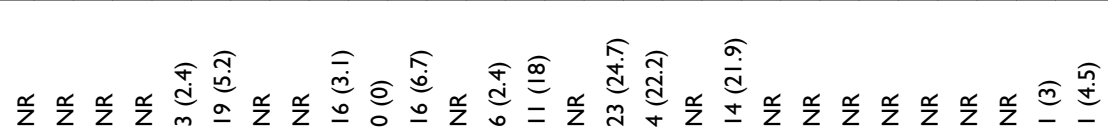 \\
\hline 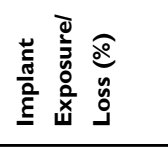 & 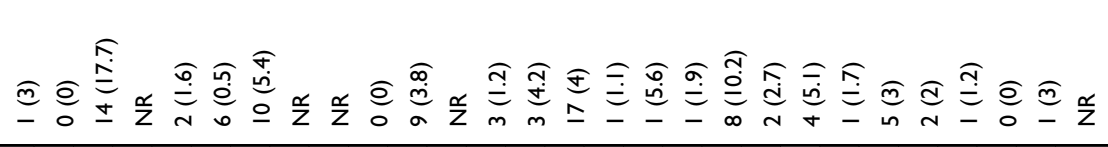 \\
\hline 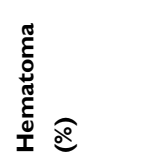 & 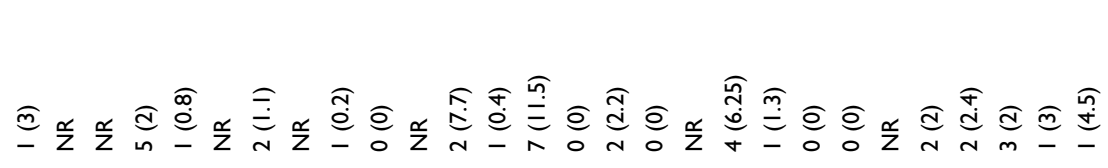 \\
\hline 气̃ & 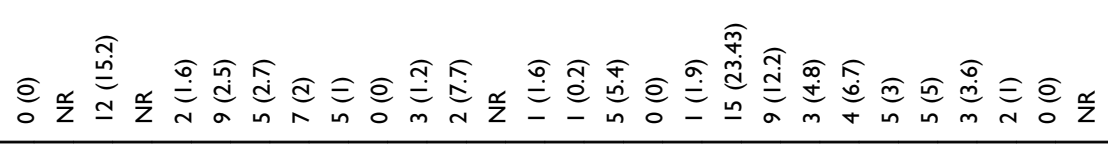 \\
\hline 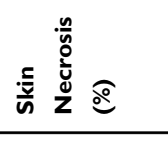 & 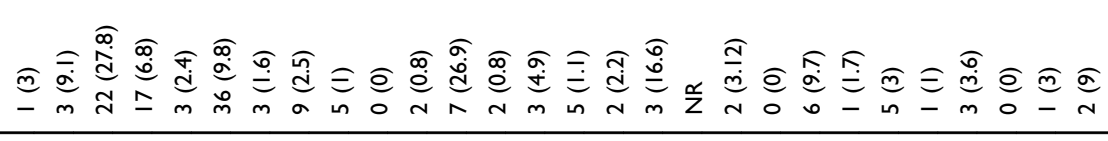 \\
\hline 莺 & 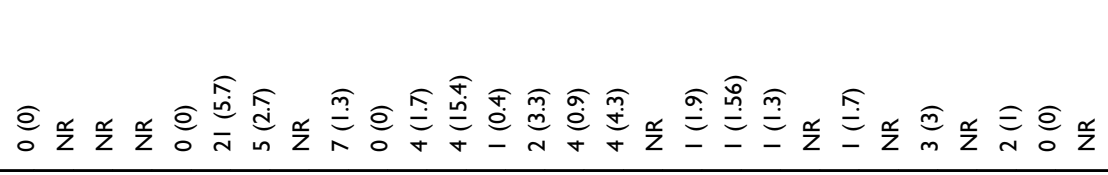 \\
\hline 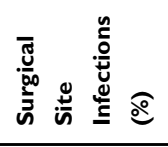 & 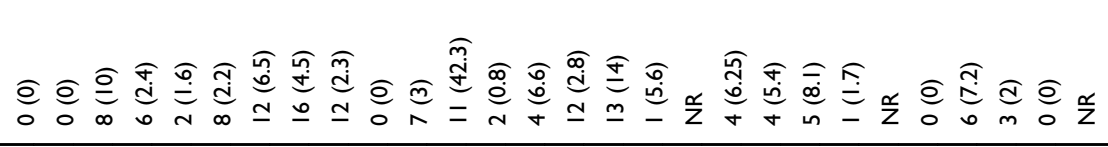 \\
\hline 竞 & 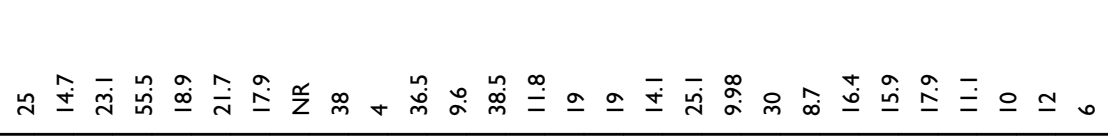 \\
\hline 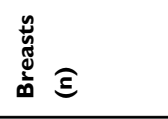 & 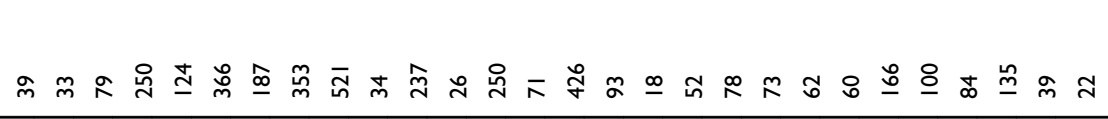 \\
\hline 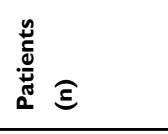 & 志え \\
\hline 商 & 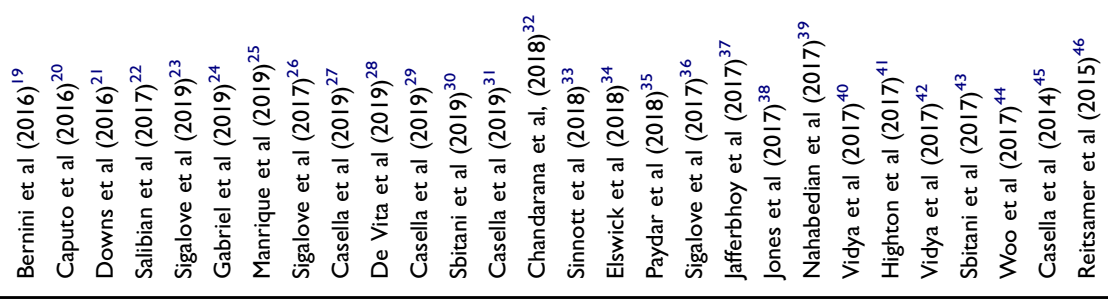 \\
\hline
\end{tabular}


articles that published various complications rates were evaluated, the results were pooled, and average complication rates were calculated as seen in Table 1. ${ }^{19-46}$

Mastectomy flap or NAC necrosis is secondary to excessive pressure on thin mastectomy tissue, impairing already disrupted perfusion. Loss of this overlying soft tissue coverage can be devastating and result in reconstruction failure. This complication can be reduced by the use of intraoperative indocyanine green tissue perfusion assessment and if any doubt exists, deferring direct-to-implant and instead opting for tissue expanders. Infection is always a concern during implant-based breast reconstruction and can lead to implant loss. The large subcutaneous pocket that is left before reconstruction opens up the possibility for hematoma or seroma formation postoperatively. Meticulous hemostasis and thoughtful drain placement help to mitigate these risks. ${ }^{15}$

Another concern is the risk of visible implant rippling at the upper pole given thinner soft tissue coverage as compared to submuscular reconstruction. During twostage prepectoral reconstruction, the tissue expanders should be underfilled relative to the anticipated final implant size in order to avoid rippling due to redundant skin during the expander-to-implant exchange operation. The rippling effect is also mitigated by planning fat grafting to the upper pole mastectomy flap during the secondstage expander-to-implant exchange operation. It is important to counsel patients who are undergoing immediate, direct-to-implant prepectoral breast reconstruction that they may need a later procedure for fat grafting over the implant to reduce rippling and implant visibility. ${ }^{3}$ Direct-to -implant breast reconstruction was in fact made possible with prepectoral implant placement as the muscle does not need to be expanded to accommodate a big implant.

\section{Effects of Radiation}

Postmastectomy radiation therapy, while lifesaving, is a known risk factor for implant-based reconstruction complications, namely capsular contracture and reconstruction failure. ${ }^{17,18}$ In a recent retrospective review, Sinnott et al reported that subpectoral breast reconstruction had a three times greater rate of capsular contracture compared to prepectoral breast reconstruction following postmastectomy radiation therapy (52.2 versus $16.1 \%$ ). Moreover, 10 of the 12 cases of capsular contracture in the subpectoral group were grades 3 or 4 compared with 2 of the 9 cases in the prepectoral group. ${ }^{17}$ It has been proposed that the increased surface area coverage of the implant by ADM is protective.
With two-stage reconstruction, expander migration is higher in the dual plane than the prepectoral group during postmastectomy radiation. This is thought to occur due to radiationinduced fibrosis and contraction of the pectoralis major muscle, which causes superior displacement of the expander. Prepectoral breast reconstruction is not subject to this phenomenon as there is no muscle coverage of the expander. ${ }^{3}$

\section{Conclusion}

Prepectoral breast reconstruction is a reliable, safe, and aesthetically feasible method of breast reconstruction following mastectomy. With the advances in implant material and ADM, capsular contracture and overall complication rates have declined. While there is no consensus on the specific technique of ADM use, both the anterior and complete coverage approaches have been used with similar success. Complications are similar to subpectoral implant placement but avoid pectoralis muscle dissection and thus shorten recovery time and improve functional outcome. Moreover, the ability to perform nipple-sparing mastectomy in combination with prepectoral breast reconstruction allows for preservation of breast form and pectoral muscle function, while providing the minimal amount of surgery. It is also important to note that prepectoral implant capsular contracture grades are lower compared to subpectoral following postmastectomy radiation therapy. That being said, the published data for prepectoral breast reconstruction outcomes have a shorter follow-up compared to their subpectoral counterpart. With more data expected to be published, plastic surgeons should continue to offer their patients reconstruction modalities that are based on the highest-quality evidence available.

\section{Disclosure}

Dr Abhishek Chatterjee reports non-financial support from Allergan, outside this submitted work. The authors report no other conflicts of interest in this work.

\section{References}

1. Patel K, Bloom J, Nardello S, Cohen S, Reiland J, Chatterjee A. An oncoplastic surgery primer: common indications, techniques, and complications in level 1 and 2 volume displacement oncoplastic surgery. Ann Surg Oncol. 2019. doi:10.1245/s10434-019-07592-5

2. Jonczyk MM, Jean J, Graham R, Chatterjee A. Surgical trends in breast cancer: a rise in novel operative treatment options over a 12 year analysis. Breast Cancer Res Treat. 2019;173(2):267-274. doi:10.1007/ s10549-018-5018-1

3. Sbitany H, Piper M, Lentz R. Prepectoral breast reconstruction: a safe alternative to submuscular prosthetic reconstruction following nipple-sparing mastectomy. Plast Reconstr Surg. 2017;140:432. doi:10.1097/PRS.0000000000003627 
4. Sigalove S, Maxwell GP, Sigalobe NM, et al. Prepectoral implant-based breast reconstruction: rationale, indications, and preliminary results. Plast Reconstr Surg. 2017;139:287. doi:10.1097/ PRS.0000000000002950

5. Ter Louw RP, Nahabedian MY. Prepectoral breast reconstruction. Plast Reconstr Surg. 2017;140:51S. doi:10.1097/PRS.0000000000003942

6. Rebowe RE, Allred LJ, Nahabedian MY. The evolution from subcutaneous to prepectoral prosthetic breast reconstruction. Plast Reconstr Surg Glob Open. 2018;6:e1797. doi:10.1097/GOX.0000000000001797

7. Spear S. Discussion: acellular dermis-assisted prosthetic breast reconstruction versus complete submuscular coverage: a head-tohead comparison of outcomes. Plast Reconstr Surg. 2009;124 (6):1741-1742. doi:10.1097/PRS.0b013e3181bf7f3a

8. Spear SL, Rottman SJ, Seiboth LA, Hannan CM. Breast reconstruction using a staged nipple-sparing mastectomy following mastopexy or reduction. Plast Reconstr Surg. 2012;129(3):572-581. doi:10.1097/PRS.0b013e318241285c

9. Antony AK, Poirier J, Madrigrano A, Kopkash KA, Robinson EC. Evolution of the surgical technique for "breast in a day" direct-toimplant breast reconstruction: transitioning from dual-plane to prepectoral implant placement. Plast Reconstr Surg. 2019;143:1547. doi:10.1097/PRS.0000000000005627

10. Vidya R, Masia J, Cawthorn S, et al. Evaluation of the effectiveness of the prepectoral breast reconstruction with Braxon dermal matrix: first multicenter European report on 100 cases. Breast J. 2017;1-7. doi: $10.1111 /$ tbj. 12810

11. Nadeem R. Prepectoral implant-based breast reconstruction; complete acellular dermal matrix wrap or anterior circumferential cover. Breast J. 2017;1-2. doi:10.1111/tbj.12881

12. Fabrizio T, Serio S, Massariello D, Vestita M, Simeon V. The meshed biological matrix in immediate, definitive breast reconstruction. JPRAS. 2019;72(1):137-171. doi:10.1016/j.bjps.2018.09.004

13. Chatterjee A, Nahabedian MY, Gabriel A, et al. Early assessment of post-surgical outcomes with pre-pectoral breast reconstruction: a literature review and meta-analysis. J Surg Oncol. 2018;117 (6):1119-1130. doi:10.1002/jso.v117.6

14. Gfrerer L, Liao EC. Technique refinement in prepectoral implant breast reconstruction with vicryl mesh pocket and acellular dermal matrix support. Plast Reconstr Surg Glob Open. 2018;6:e1749. doi:10.1097/GOX.0000000000001749

15. Wagner RD, Braun TL, Zhu H, Winocour S. A systematic review of complications in prepectoral breast reconstruction. J Plast Reconstr Aesthetic Surg. 2019;72:1051-1059. doi:10.1016/j.bjps.2019.04.005

16. Nahabedian MY. Prosthetic breast reconstruction and red breast syndrome: demystification and a review of the literature. Plast Reconstr Surg. 2019;7:e2108. doi:10.1097/GOX.0000000000002108

17. Sinnott CJ, Persing SM, Pronovost M, Hodyl C, McConnell D, Young AO. Impact of postmastectomy radiation therapy in prepectoral versus subpectoral implant-based breast reconstruction. Ann Surg Oncol. 2018;25(10):2899-2908. doi:10.1245/s10434-018-6602-7

18. Elswick SM, Harless CA, Bishop SN, et al. Prepectoral implant-based breast reconstruction with postmastectomy radiation therapy. Plast Reconstr Surg. 2018;142:1. doi:10.1097/ PRS.0000000000004453

19. Bernini M, Calabrese C, Cecconi L, et al. Subcutaneous direct-toimplant breast reconstruction: surgical, functional, and aesthetic results after long-term follow-up. Plast Reconstr Surg Glob Open. 2016;3(12):e574. doi:10.1097/GOX.0000000000000533

20. Caputo GG, Marchetti A, Dalla Pozza E, et al. Skin-reduction breast reconstructions with prepectoral implant. Plast Reconstr Surg. 2016;137(6):1702-1705. doi:10.1097/PRS.0000000000002227

21. Downs RK, Hedges K. An alternative technique for immediate direct-toimplant breast reconstruction-a case series. Plast Reconstr Surg Glob Open. 2016;4(7):e821. doi:10.1097/GOX.0000000000000839.
22. Salibian AH, Harness JK, Mowlds DS. Staged suprapectoral expander/implant reconstruction without acellular dermal matrix following nipple-sparing mastectomy. Plast Reconstr Surg. 2017;139(1):30-39. doi:10.1097/PRS.0000000000002845

23. Sigalove S, Maxwell GP, Gabriel A. Utilizing a spectrum of cohesive implants in aesthetic and reconstructive breast surgery. Plast Reconstr Surg. 2019;144(1S):66S-72S. doi:10.1097/PRS.0000000000005952

24. Gabriel A, Sigalove S, Sigalove N, et al. Effect of body mass index on outcomes after prepectoral breast reconstruction. Plast Reconstr Surg. 2019. doi:10.1097/PRS.0000000000005901

25. Manrique OJ, Banuelos J, Abu-Ghname A, et al. Surgical outcomes of prepectoral versus subpectoral implant-based breast reconstruction in young women. Plast Reconstr Surg Glob Open. 2019;7(3):e2119. doi:10.1097/GOX.0000000000002119.

26. Sigalove S, Maxwell GP, Sigalove NM, et al. Prepectoral implant-based breast reconstruction: rationale, indications and preliminary results. Plast Reconstr Surg. 2017;139:287-294. doi:10.1097/ PRS.0000000000002950

27. Casella D, Di Taranto G, Onesti MG, Greco M, Ribuffo D. A retrospective comparative analysis of risk factors and outcomes in direct-to-implant and two-stages prepectoral breast reconstruction: BMI and radiotherapy as new selection criteria of patients. Eur J Surg Oncol. 2019;45(8):1357-1363. doi:10.1016/j.ejso.2019.02.009.

28. De Vita R, Buccheri EM, Villanucci A, Pozzi M. Breast reconstruction actualized in nipple-sparing mastectomy and direct-to-implant, prepectoral polyurethane positioning: early experience and preliminary results. Clin Breast Cancer. 2019;19(2):e358-e363. doi:10.1016/j.clbc.2018.12.015.

29. Casella D, Di Taranto G, Marcasciano M, et al. Subcutaneous expanders and synthetic mesh for breast reconstruction: long-term and patient-reported BREAST-Q outcomes of a single-center prospective study. J Plast Reconstr Aesthet Surg. 2019;72(5):805-812. doi:10.1016/j.bjps.2018.12.018.

30. Sbitany H, Gomez-Sanchez C, Piper M, Lentz R. Prepectoral breast reconstruction in the setting of postmastectomy radiation therapy: an assessment of clinical outcomes and benefits. Plast Reconstr Surg. 2019;143(1):10-20. doi:10.1097/PRS.0000000000005140

31. Casella D, Di Taranto G, Marcasciano M, et al. Evaluation of prepectoral implant placement and complete coverage with TiLoop bra mesh for breast reconstruction: a prospective study on long-term and patient-reported BREAST-Q Outcomes. Plast Reconstr Surg. 2019;143(1):1e-9e. doi:10.1097/PRS.0000000000005078

32. Chandarana MN, Jafferbhoy S, Marla S, Soumian S, Narayanan S. Acellular dermal matrix in implant-based immediate breast reconstructions: a comparison of prepectoral and subpectoral approach. Gland Surg. 2018;7(Suppl 1):S64-S69. doi:10.21037/gs.2018.03.05

33. Sinnott CJ, Persing SM, Pronovost M, Hodyl C, McConnell D, Ott Young A. Impact of postmastectomy radiation therapy in prepectoral versus subpectoral implant-based breast reconstruction. Ann Surg Oncol. 2018;25(10):2899-2908. doi:10.1245/s10434-018-6602-7.

34. Elswick SM, Harless CA, Bishop SN, et al. Prepectoral implant-based breast reconstruction with postmastectomy radiation therapy. Plast Reconstr Surg. 2018;142(1):1-12. doi:10.1097/ PRS.0000000000004453

35. Paydar KZ, Wirth GA, Mowlds DS. Prepectoral breast reconstruction with fenestrated acellular dermal matrix: a novel design. Plast Reconstr Surg Glob Open. 2018;6(4):e1712. doi:10.1097/ GOX.0000000000001712.

36. Sigalove S, Maxwell GP, Sigalove NM, et al. Prepectoral implant-based breast reconstruction and postmastectomy radiotherapy: short-term outcomes. Plast Reconstr Surg Glob Open. 2017;5 (12):e1631. doi:10.1097/GOX.0000000000001631.

37. Jafferbhoy S, Chandarana M, Houlihan M, et al. Early multicentre experience of pre-pectoral implant based immediate breast reconstruction using Braxon ${ }^{\circledR}$. Gland Surg. 2017;6(6):682-688. doi:10.21037/gs.2017.07.07 
38. Jones G, Yoo A, King V, et al. Prepectoral immediate direct-toimplant breast reconstruction with anterior AlloDerm coverage. Plast Reconstr Surg. 2017;140(6SPrepectoral Breast Reconstruction):31S-38S. doi:10.1097/PRS.0000000000004048

39. Nahabedian MY, Cocilovo C. Two-stage prosthetic breast reconstruction: a comparison between prepectoral and partial subpectoral techniques. Plast Reconstr Surg. 2017;140(6SPrepectoral Breast Reconstruction):22S-30S. doi:10.1097/PRS.0000000000004047

40. Vidya R, Cawthorn SJ. Muscle-sparing ADM-assisted breast reconstruction technique using complete breast implant coverage: a dual-institute UK-based experience. Breast Care (Basel). 2017;12 (4):251-254. doi:10.1159/000464401.

41. Highton L, Johnson R, Kirwan C, Murphy J. Prepectoral implant-based breast reconstruction. Plast Reconstr Surg Glob Open. 2017;5(9):e1488. doi:10.1097/GOX.0000000000001488.

42. Vidya R, Masià $\mathrm{J}$, Cawthorn $\mathrm{S}$, et al. Evaluation of the effectiveness of the prepectoral breast reconstruction with Braxon dermal matrix: first multicenter European report on 100 cases. Breast J. 2017;23 (6):670-676. doi:10.1111/tbj.12810
43. Sbitany H, Piper M, Lentz R. Prepectoral breast reconstruction: a safe alternative to submuscular prosthetic reconstruction following nipple-sparing mastectomy. Plast Reconstr Surg. 2017;140 (3):432-443. doi:10.1097/PRS.0000000000003627

44. Woo A, Harless C, Jacobson SR. Revisiting an old place: single-surgeon experience on post-mastectomy subcutaneous implant-based breast reconstruction. Breast J. 2017;23(5):545-553. doi:10.1111/tbj.12790.

45. Casella D, Bernini M, Bencini L, et al. TiLoop ${ }^{\circledR}$ Bra mesh used for immediate breast reconstruction: comparison of retropectoral and subcutaneous implant placement in a prospective single-institution series. Eur J Plast Surg. 2014;37(11):599-604. doi:10.1007/s00238014-1001-1.

46. Reitsamer R, Peintinger F. Prepectoral implant placement and complete coverage with porcine acellular dermal matrix: a new technique for direct-to-implant breast reconstruction after nipple-sparing mastectomy. J Plast Reconstr Aesthet Surg. 2015;68(2):162-167. doi:10.1016/j.bjps.2014.10.012.
Open Access Surgery

\section{Publish your work in this journal}

Open Access Surgery is an international, peer-reviewed, open access journal that focuses on all aspects of surgical procedures and interventions. Patient care around the peri-operative period and patient outcomes post-surgery are key topics for the journal. All grades of surgery from minor cosmetic interventions to major surgica procedures are covered. Novel techniques and the utilization of new

Submit your manuscript here: https://www.dovepress.com/open-access-surgery-journal

\section{Dovepress}

instruments and materials, including implants and prostheses that optimize outcomes constitute major areas of interest. The manuscript management system is completely online and includes a very quick and fair peer-review system, which is all easy to use. Visit http://www.dovepress.com/testimonials.php to read real quotes from published authors. 